\title{
Analyzing and dating the structure of the Phaistos Disk
}

\author{
Wolfgang Reczko
}

Received: 2 February 2009 /Accepted: 4 August 2009/Published online: 12 September 2009

(C) Springer-Verlag 2009

\begin{abstract}
This paper offers an analysis and dating of the structure of the "Phaistos Disk", made from clay, imprinted with undeciphered symbols, grouped in roughly 30 boxes per disk side, and found in the ruins of the Phaistos Palace on the Greek Island of Crete by Luigi Pernier in 1908. The author suggests that the disk side A containing a petal in the center box shows astronomical eclipse information, which belong to a complete Saros cycle beginning -1377 and valid for the Phaistos Palace location only.
\end{abstract}

Keywords Phaistos Disk $\cdot$ Structure $\cdot$ Saros $\cdot$ Crete

\section{Introduction}

The Phaistos Disk is a circular clay disk of $16 \mathrm{~cm}$ diameter and $1 \mathrm{~cm}$ thickness. It was excavated by Luigi Pernier in 1908 in the ruins of Phaistos Palace, Crete and was dated as follows:

Sir A. Evans (1921) suggests reading direction from center

Dr. Thomas Balistier (1998): "Der Diskos von Phaistos", p 36

Yves Duhoux (1977) dates the disk between $1850 \mathrm{BC}$ and $1600 \mathrm{BC}$

Louis Godart (1995) dates it to anywhere in Middle/ Late Minoan

The disk's mystery is its symbols, grouped in total of 61 boxes on both disk sides (Balistier 1998).

W. Reczko $(\bowtie)$

Bahnhofstr. 26,

71088 Holzgerlingen, Germany

e-mail: 1reczko@arcor.de
Although many symbols look like pictorial images of everyday life - a walking human, a fish, a flying bird - the disk's "message" has not yet (July 2009) been deciphered; there have been many suggestions, but none has been found valid and approved.

One main cause may be a missing second object with similar symbols in the same "alphabet" to compare to the disk's symbols: Apart from some broken artifacts with very few symbols, no object has been found with a comparable count of symbols - more than the disk's 241 -in the world.

Consequently, it is currently not very reasonable adding another deciphering to the attempted ones.

Therefore, this paper concentrates on the structure of the boxes holding the symbols, which may be compared to other structures of today or of the past that are known today, e.g., National Aeronautics and Space Administration (NASA) celestial eclipse calculations (see step 5.3 below).

This paper aims at suggesting the type of structure, the geographic place of design, a partial purpose of the disk, and the date its design represents.

\section{Description of the Phaistos Disk and eclipse lists (for a picture of both disk sides, see Thomas Balistier 1998)}

There exists a total of 241 symbols on the whole disk (A + B sides), selected from a pool of 45 unique symbols, numbered by Sir Arthur Evans (1921, Appendix 1), who also defined the disk side A as having a flower petal in the center.

These 241 symbols are grouped in boxes (average of four in one box): There are 31 boxes on disk side $\mathrm{A}$ and 30 on side $\mathrm{B}$.

Each disk side has the same structure of boxes: Both sides have an inner group in spiral form and an outer group on the disk's rim. The rim of both sides has 12 boxes. 
The spiral of both sides has 18 boxes with a small but important box on side B right after the 18th box counted from the center. The symbol count of two in this little box deviates obviously from the average of four (see "Analysis and suggestions" section below).

In order to work with the boxes, the following naming is introduced in this paper, which easily relates to this paper's analysis ("Appendix 3 and 5"). In naming the boxes, each box is identified by three components:

1. The disk side: A and B for sides A and B, respectively

2. The group: $\mathrm{s}$ and $\mathrm{r}$ for spiral and rim, respectively

3. The position: $01 \ldots 18$ for the inner spiral and $01 \ldots 12$ for the outer rim

Example: The spiral's center box of disk side $A=A s 01$. Note that there is a small box with only two symbols following spiral box As18, named As18b (see "Analysis and suggestions" section and Appendix 2 below).

This structure is not visible on first glance, e.g., both box groups, the inner spiral and the outer rim, were often identified as one contiguous group, as can be seen in the literature, where the side B's box names ranged from B01not stopping at B18 - to B30; obviously, the short break at B18 is overlooked, and the possibility to identify a Saros cycle is missed.

Without this 18 -year cycle, the chance is missed to identify the dimension of all boxes as time elements (one box=1 year, except small box As18b, see the first step under "Analysis and suggestions" section). Two sources of information were needed for analysis: the detailed description of the Phaistos Disk (see Balistier Thomas 1998) and the precise eclipse lists from Fred Espenak, Goddard Space Flight Center (GSFC)/NASA (see Espenak 2009).

\section{Analysis and suggestions}

The first general step is to associate the inner box spiral to the astronomical eclipse cycle Saros of roughly 18 years.

The second step is to identify some of the disk spiral boxes as having duplicates somewhere on the same spiral, building a pattern of associated boxes.

After detecting a certain pattern of duplicate boxes, the idea is to search the available lists of ancient eclipses forin the best case - a single match with the disk's specific pattern, which is the third step.

This analysis tries to shed some light on the design of the disk and the geographic location of its astronomic reference. To verify the eclipse-matching method, the author assigned an intentionally wrong geoposition as input (also on Crete) and received no matching pattern as expected; this result also reduces the possibility of a design outside Crete.

Please note that this paper does not state the date or location of the disk's manufacturing, although it may be assumed to be on Crete, after its Cretan design.

The first step is identifying the Saros cycle principle, i.e., the reason for picking the first 18 boxes from the "center" of the disk side A (with the flower petal in the center) is the similarity of this box count to the 18 years of the astronomic Saros cycle (more exact=18 years, 11 days, $8 \mathrm{~h}$ ). Another crucial reason for picking the 18 boxes (here, of side A) is the small box As18b at the end of the side A spiral, starting at box As01 in the center, following the spiral outward to box As18, and finding a small box (two symbols only) behind box As18, which fits to account for the Saros period rest of 11 days $8 \mathrm{~h}$ as it signals the end of Saros cycle and the end of the box spiral.

These are some of the reasons to name it box As18b, not As19, and the assumption of the center box As01, being the start of the box spiral, confirms the first assumption of Sir A. Evans (1921). A second try of pattern matching was done using the inverted disk box spiral (not center box first, but 18th box first): The resulting lack of matching patterns indicated that the first method (start of reading in the center) seems to be better, also confirming the reading method of Sir A. Evans (1921).

This is an important identification in this paper which led to the astronomical cycle "Saros" ("Appendix 3"), building a bridge between archeology and astronomy and refining this analysis' path.

The 12 boxes on the rim of the disk are not considered in detail in this paper. Still, it could be assumed-in the wake of the spiral box being an astronomic object - that the number 12 may suggest the astronomic 12 months of a year, commonly used in some cultures. Thus, the 12-rim boxes could represent any year-yet unknown. The second step to take is identifying the year duplicates on the disk spirals in order to solve the problem of associating one Phaistos Disk spiral to all relevant ancient Saros cycles.

List of duplicates found on disk sides A and B:

Side A

Four twins

Boxes As01 and As04 (flower petal, head + shovel)

Boxes As03 and As15 (comb, two plants, two hides, cookie, irokese)

Boxes As11 and As17 (Walker, Stick)

Boxes As12 and As18 (boomerang + five others)

One triplet: Boxes As10, As13 and As16 (Eel, Bird, Cookie, Irokese) 


\section{Side B}

One twin

Boxes Bs05 and Bs10 (glove, + three others), i.e., only one twin: one reason for side $\mathrm{B}$ not taking part in identifying the beginning of the final Saros cycle. See also Appendix 6.

The third and final step is analyzing the matching eclipses and interpreting the best match of the disk's "Saros" cycle/spiral. Here, it is assumed that the duplicate (+ triplet) years of the second step above must have something in common; the hypothesis used is if the whole disk spiral represents a Saros cycle with eclipses, then the "twin" events in twin years can be assumed to represent reoccurring Sun eclipses of 2 years (twin) or 3 years (triplet).

There is one twin (As03, As15) where no Sun eclipse occurred in the same Saros period (spiral of side A). This gap is filled by extracting the Moon eclipses of this same Saros period: There appeared Moon eclipses in the position As03 and As15, as expected, which verified the previous Sun eclipse matching. A manual procedure ("Appendix 3", Selecting the best match of two Saros cycles) was developed for searching a contiguous number of 500 years (see below the limits), trying to find all 18-year periods, which fulfill the twin and triplet pattern extracted from the disk (from above second step).

The second input is primarily the Sun eclipse list produced by the GSFC/NASA for the period -1500 to -1000 : The year limit of -1500 is the minimal limit allowed by the GSFC designer of this tool; the upper/youngest limit -1000 is chosen for making sure to cover the suggestions of Godart, who assigns the disk to the time between -1500 and thirteenth century BC (Godart 1995, p 162; Balistier 1998, p 27).

The geographic position of the Phaistos Palace $-24^{\circ} 48^{\prime} \mathrm{E}$, $35^{\circ} 05^{\prime} \mathrm{N}$ - is used to generate 500 contiguous years of ancient eclipses. This geoposition is used twice for this paper:

1. To generate the pattern lists mentioned above under "Analysis and suggestions" section

2. To verify the assumed location "Phaistos" as the geoposition of the disk designer by varying the coordinates; this leads, as expected, to a nonmatching pattern list.

Example: The eclipse list for, e.g., Phaistos, Crete results in following eclipse list, assuming otherwise identical parameters (e.g., century).

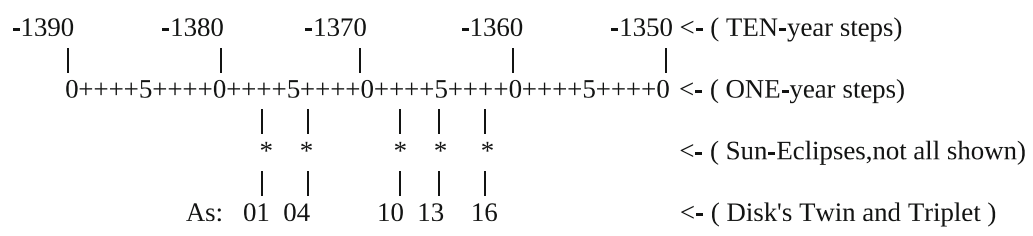

There are five matches (in years $-1377,-1374,-1368$, -1365 , and -1362). These 500 years are taken as a time base, and the second input (18 years with the eclipse twins and triplet, see step 2 above) is plotted stepping through the 500 years, each year, checking for a best match.

Chosen for pattern matching were:

Side A

1 triplet-unit (covering 7 Saros-years: $10 \ldots 16$

As10/As13/As16

1 twin-unit (covering 4 Saros-years: $01 \ldots 04$

As01/As04

Sum of covered years: $7+4=11$ from total 18

Span of covered years: As01...As16 $=16$ years of 18 .

Note that the twins between years 11 and 18 were initially left out because they mostly overlapped the triplet, which itself provides far better result singularity.
There is only one optimal match with the selected twin/ triplet combination with maximum eclipse magnitude: The year -1377 (5th of July, beginning 0501 hours) suggested as the initial year of a Saros cycle starting in year -1377 , extending to -1359 . To enhance the result, the disk's twin of the same period (year twin As03 and As15) can be found in the same Moon eclipse list for the same Sun eclipse period starting -1377 on the NASA list and - reassuringthe second Sun eclipse documented by the Phaistos Disk/ year box As04 = eclipse list year -1374 was also independently observed by Ugarit astronomers in year 1375 BC (=-1374 BC, see "Appendix 4").

There is a second triplet/twin match of Sun eclipses, but the magnitude of the first eclipse in -1377 was higher $(100 \%)$, and it occurred in the last century of the five probed, -1023 (see "Appendix 3", cutout). To further assess the significance of the optimal match, each of the relevant duplicated boxes is systematically omitted, and the 
Table 1 Analysis of match significance using systematic omissions of the relevant duplicated boxes

\begin{tabular}{lcll}
\hline Box suppressed & Count & Matching year & Magnitude $\times 10$ \\
\hline As01 & 3 & 1377,1374 & 7,10 \\
& & 1314 & 5 \\
As04 & 8 & 1468,1452 & 9,5 \\
& & 1440,1398 & 3,6 \\
& & 1377,1374 & 7,10 \\
& & 1076,1023 & 4,4 \\
As10 & 5 & 1389,1377 & 7,7 \\
& & 1351,1334 & 6,9 \\
& & 1023 & 4 \\
As13 & 4 & 1478,1377 & 4,7 \\
& & 1304,1023 & 3,4 \\
As16 & 5 & 1440,1386 & 3,5 \\
& & 1377,1032 & 7,6 \\
\hline
\end{tabular}

Average count of matches $=5$; standard deviation $=1.67$

resulting matches are listed in Table 1. In all of these cases, an obviously higher number of matching years, averaging 5 ( \pm 1.67$)$, emphasizes the significance of the optimal match.

\section{Conclusion}

This paper suggests that the main structure side A of archeological Phaistos Disk found on Crete in 1908 represents the astronomical Saros cycle beginning year -1377 ; it is indicated by matching the disk's associated duplicates with eclipse lists from GSFC/NASA (Fred Espenak). The knowledge needed for creating this Phaistos Disk - the rhythm of the 18+years of the Saros Cycle-is assumed to have enabled the creator(s) predicting future eclipses.

Acknowledgments The initial idea to analyze the Phaistos Disk stems was from Dr. M. Reczko in 2007, working in the FORTH Institute in Heraklion, Crete. This paper would have taken much longer to create (if ever) would it not have been for the "Eclipse Predictions by Fred Espenak and Chris O'Byrne (NASA's GSFC)”, copyright 2008.

\section{Appendix 1}

Numbering the disk symbols The archeologist Sir Arthur Evans has numbered the 45 symbol types from 1 to 45 (starting at the center). This paper follows these numberings and uses them - concatenated - to form an identification key for each occurrence of 1-year box. This saves time when sorting all the 61-year boxes in order to find duplicate boxes fast and correct instead of sorting them "manually".

\section{Appendix 2}

There is one small box $18 \mathrm{~b}$ at the end of the side A spiral, in an inhomogeneous position and form behind box As18, which is difficult to place, but if the exact length of a Saros cycle is taken ( 18 years +11 days $+8 \mathrm{~h}=18.032$ years; please mind the decimal, not 18,000 and 3 years), then this box $18 \mathrm{~b}$ could account for the rest of 0.032 years.

Also interesting to see is that disk side $\mathrm{B}$, which is found to represent 18 years earlier than side $\mathrm{A}$, is missing the small, more precise box $18 \mathrm{~b}$.

This could give rise to assume an increase of knowledge of the disk's creators during their development.

This assumption is encouraged when comparing the "finish" and homogenous arrangement and information of all boxes of side A to side B: The side A arrangement is not only more geometric but it also contains informative 11 duplicate years compared to two duplicate years on side B.

\section{Appendix 3}

Saros cycle

A Saros cycle is a period of about 18 years $(+11$ days $+8 \mathrm{~h})$. After this period, the eclipses of Sun/Moon are again in phase (repeating same constellation but $8 \mathrm{~h}=120^{\circ} \mathrm{W}$ of first position). After three cycles $\left(3 \times 8 \mathrm{~h}=24 \mathrm{~h}\right.$ or $\left.360^{\circ}\right)$, the eclipses occur (roughly) in the same Earth region.

Because of other gravitational pull, the Moon's path is slowly offset (about 1,000 years for a complete cycle called Saros series, not cycle), moving its shadow on Earth - in addition to the east-west direction - from south to north and back, introducing a long-term offset until same Earth-SunMoon constellation could repeat. It follows that our eclipse combination of, e.g., -1377 , reoccurs much later (about -377 ) than the ancient history of the mideast in these periods, increasing the chance of singularity of Saros-year -1377 .

Selecting the best match of two Saros cycles

\section{General}

There was a second "best-match" in the century $-1100 \ldots$ -1000 , and shown below is the reason for picking year -1377 as start of the Saros cycle depicted on the Phaistos Disk: The magnitude of the eclipse in -1374 was higher than any other in candidate Saros beginning in year -1023 . This finding fits the period suggested by Louis Godart. 


\section{Details}

Legend:

Eclipse magnitude (magnitude $1.0=100 \%$ of Sun's diameter shadowed)

Eclipse obscuring (obscuring 1.0 $=100 \%$ of Sun's area obscured)

Candidate Saros cycle in century -1400 to -1300 (shown here is the cutout of 40 years from century -1400 to -1300 ):

Picked years $=-1390$ to -1350 with best match: begins -1377
Eclipses from NASA are shown below the year scale in the form of month, e.g., eclipse in year-1390/September shown as "9" (months January... October...December $\Rightarrow$ $1 \ldots \mathrm{a} \ldots \mathrm{c})$

Below are:

- The year scale (two lines)

- The eclipse scale (one line)

- The Saros duplicate years side A (As01, As04, As10, As13, AS16), with As01 positioned under best match year -1377

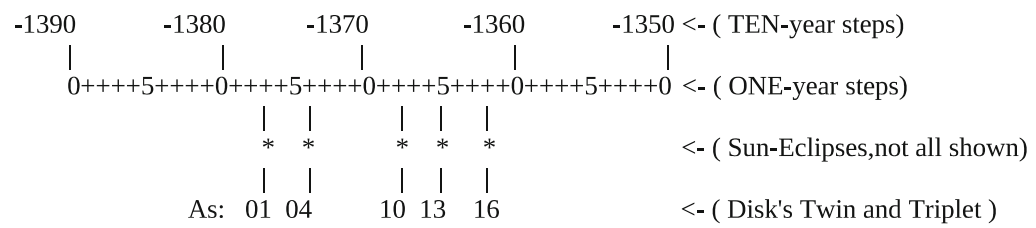

Second of two candidate Saros cycles in century -1100 to -1000 :

40 years $=$ candidate match: begins -1023 but no magnitude/obscurence reached the value $1.0=$ total

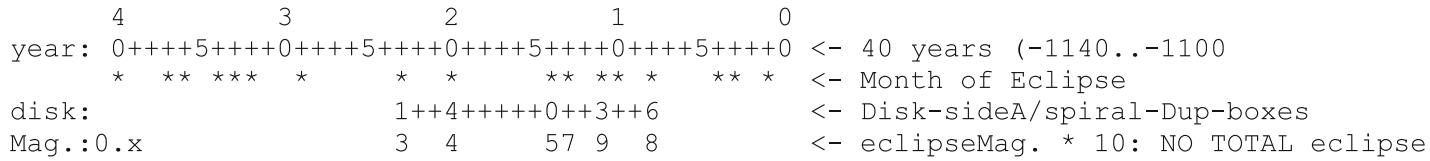

\section{Appendix 4: Date format}

Please note a "1"-year difference in date formats: Conventional date format of ancient objects is left unchanged (Ugarit Sun eclipse 1375 BC); also left unchanged are all eclipse dates from GSFC/NASA with a minus sign (same Ugarit Sun eclipse -1374). So, it is normal if some eclipse dates from other - conventional-sources/books are shifted by 1 (example, Ugarit Sun eclipse dating, "1375 BC"-1=“-1374").

\section{Appendix 5}

Names of disk's boxes I named the disk boxes as shown at the end of step 1 in order to fit my approach best.

\section{Appendix 6}

The Moon eclipses occur more homogenous in the Moon eclipse list than the Sun eclipse list. Therefore, the Sun eclipse list was selected as the main pattern input; only when twin As03/As15 had no Sun eclipse match that the Moon eclipse list was - successfully — used as a match.

\section{References}

Balistier T (1998) Der Diskos von Phaistos. Balistier, Muhringen, p 36

Evans AJ (1921) Palace of Minos: "It might, a priori, have been supposed that the signs of the inscriptions had run outwards,", vol 1. Macmillan, London, p 649

Godart L (1995) Der Diskos von Phaestos. ITANOS, Athens

Espenak F et al (2009) Web pages with the sun/moon-eclipse lists (historical structures) of Goddard Space Flight Center/NASA (copyright by Fred Espenak et al., GSFC/NASA); URL dated 19 Jan 2009: http://eclipse.gsfcnasa.gov/solar.html "Javascript Solar Eclipse Explorer" (needs "JavaScript=ON" in browser)

Duhoux Y (1977) Le Disque de Phaistos. Peeters, Louvain 